\title{
Gestión de la innovación social cooperativa a través de una cultura solidaria innovadora
}

Alveiro Monsalve Zapata*

Magíster en Educación. Profesor, Pontificia Universidad Javeriana, Bogotá, Colombia. Consultor de diferentes organizaciones solidarias en Colombia. Correo electrónico: alveiro.monsalve@ javeriana.edu.co

Recibido: 17 de marzo del 2015

Aceptado: 9 de noviembre del 2015

Cómo citar este artículo: MonsalveZapata, A. (2015). Gestión de la innovación social cooperativa a través de una cultura solidaria innovadora. Cooperativismo y Desarrollo, 24(108), 27-38. doi: http://dx.doi. org/10.16925/co.v24i108.1258

\section{Resumen}

Propósito: el propósito de este artículo es ofrecer una mirada rápida a las posibilidades de la innovación social en el contexto de la cultura solidaria que debe caracterizar a las organizaciones cooperativas o similares. Descripción: con base en investigaciones de diferentes autores se definen y relacionan entre sí los conceptos de innovación social y cultura de la solidaridad, desde las perspectivas antropológica, sociológica, económica y cooperativa. Punto de vista: se exploran posibilidades de cambio en la cultura solidaria de las empresas cooperativas a través de la gestión específica en el campo de la innovación social. Conclusión: mediante una nueva pedagogía social es posible transformar la cultura solidaria actual en una dinámica experiencia de innovación social.

Palabras clave: cooperativismo, cultura solidaria, economía social, innovación social. 


\title{
Management of Social-Cooperative Innovation through an Innovative Culture of Solidarity
}

\begin{abstract}
Purpose: This article aims to provide an overview of the possibilities of social innovation in the context of a culture of solidarity that should characterize cooperatives or similar organizations. Description: Based on research by different authors, the concepts of social innovation and culture of solidarity are defined and interrelated from anthropological, sociological, economic and cooperative perspectives. Point of view: Possibilities for change are explored in the cooperatives' culture of solidarity through specific management in the field of social innovation. Conclusion: Using a new social pedagogy, it is possible to transform the current culture of solidarity into a dynamic experience of social innovation.
\end{abstract}

Keywords: cooperative movement, culture of solidarity, social economy, social innovation.

\section{Gestão da inovação social-cooperativa por meio de uma cultura solidária inovadora}

\section{Resumo}

Propósito: este artigo se propõe oferecer um breve olhar nas possibilidades da inovação social no contexto da cultura solidária que deve caracterizar as organizações cooperativas ou similares. Descrição: com base em pesquisas de diferentes autores, definem-se e relacionam-se entre si os conceitos de inovação social e cultura da solidariedade, sob as perspectivas antropológica, sociológica, econômica e cooperativa. Ponto de vista: exploram-se possibilidades de mudança na cultura solidária das empresas cooperativas por meio da gestão específica no campo da inovação social. Conclusão: mediante uma nova pedagogia social, é possível transformar a cultura solidária atual numa dinâmica experiência de inovação social.

Palavras-chave: cooperativismo, cultura solidária, economia social, inovação social. 


\section{Introducción}

Mucho se discute en diferentes ámbitos de pensamiento sobre las razones que explican la falta de incidencia de las cooperativas en el entorno inmediato de las comunidades que las rodean.

Hay muchos factores a tener en cuenta, entre ellos la carencia de una verdadera cultura solidaria que fortalezca la identidad del modelo cooperativo, el sentido de pertenencia, la participación amplia y la sostenibilidad del grupo humano que constituye la asociación cooperativa.

Parecería que es más importante fortalecer primero la conciencia solidaria, antes que desarrollar procesos pedagógicos de educación para la solidaridad. Si hay verdadera cultura solidaria habrá procesos sostenibles de cooperación asociativa, de lo contrario no.

Este es uno de los grandes retos entre quienes dirigen o lideran el desarrollo de cualquier cooperativa. Y tanto más grande será el reto, si la responsabilidad social cooperativa desde la visión empresarial, se convierte en procesos creativos de innovación social, de tal manera que pueda ser más eficaz la incidencia que reclaman tantas comunidades en el mundo a través del movimiento cooperativo.

\section{Cultura e innovación como estrategia histórica}

Siempre será de máxima importancia en el movimiento cooperativo profundizar sobre la gestión de la innovación social y en la creación de una nueva cultura solidaria, como aspectos que deben caracterizar el clima ético y moral (Martínez, 2005) de cualquier empresa cooperativa por pequeña o grande que sea.

Al reflexionar sobre la naturaleza sociológica y antropológica (Weber, 2011) de la cultura de la solidaridad en el ambiente asociativo, surge también la idea de si ella hace parte vital o secundaria de la ventaja estratégica cooperativa que es en sí misma un modelo de innovación social.

La naturaleza viva que nos rodea, es una insuperable maestra de la innovación. En ella surgió el hombre, modelo singular de permanente innovación (Goleman, Kaufman y Ray, 2000).

Desde el Mioceno, hace tres millones de años según los paleontólogos, comienza nuestra historia de homínidos bípedos, con capacidad de innovación. Fueron muchas y misteriosas las mutaciones para que el australopitecus llegara a caminar en dos pies, tener libres sus extremidades superiores y poder así ahorrar mucha energía en sus desplazamientos o cargar cosas sobre sus hombros y otear a lo lejos con más facilidad.

El homo erectus hace unos dos millones de años, dio paso al homo hábilis, que usaba ya las manos libres y podía entonces con su cerebro innovador, manejar herramientas muy elementales que le sirvieran para modificar su entorno y defenderse.

En el paleolítico, el homo sapiens con su complejo sistema nervioso central ya era capaz de innovar en la construcción de sus rústicas viviendas y fabricar herramientas con piedras y maderas. Pero sobre todo, aprendió a comunicarse por medio del lenguaje hablado y lo hizo de una forma tal, que se diferenció así del resto de animales. El lenguaje humano lo convirtió en un ser universal.

Para comunicarse entre sí, el homo sapiens se volvió un animal generador de símbolos (Guiraud, 1996). En el mundo de los símbolos hay un poder mágico para hacer gestión de la innovación social en cualquier contexto cultural. ¡No se puede concebir el pensamiento humano sin la semiótica de los símbolos! Símbolos que no siempre se comprenden entre la diversidad cultural que desde siempre ha caracterizado a la civilización humana.

Con el lenguaje y con los símbolos, en medio de la convivencia colectiva (Weber, 2011), nació la cultura: un conjunto abigarrado de conductas y costumbres, de maneras de ser, de verdades y mentiras, de morales inventadas por los dueños del poder, de artilugios religiosos y de ideas oscuras mezcladas con pensamientos luminosos (Guiraud, 1996). En este proceso civilizador que ha durado milenios, siempre ha sido necesaria la innovación como sustento creativo de la cultura.

En medio de este proceso evolutivo (Schnarch, 2006) aparece también el homo faber, el homo innovador, el gestor y constructor de la ciencia y de la tecnología, el creador de nuevos mundos a partir de su mente y su cerebro. Pero también y a pesar de todo, este mismo homo faber es capaz de hacer la guerra contra todos sus congéneres o de cometer actos bárbaros y absurdos o dejarse llevar por el afán corrosivo del egoísmo individual o manejar además el poder, cualquiera que sea, con absoluta irracionalidad. Así se crearon los imperios, con el arrollador poderío de las innovaciones militares.

\section{El poder creativo de la mente humana}

Es pues la capacidad de innovar, un atributo de la mente humana, una categoría elevada en niveles de 
su propia conciencia, una fase superior del universo (Goleman et al., 2000). El poder creativo del hombre, alimentado por el conocimiento y la curiosidad, se convierte en fuente inagotable del saber, en la que la imaginación no tiene límites (Vélez y Vélez, 2010). Capacidad de innovar aplicada al bien o aplicada al mal. He aquí, en esta última afirmación, el componente ético de la innovación (Martínez, 2005).

Gracias a esta facultad de su intelecto, el ser humano hace ciencia y tecnología, amplía cada vez más las fronteras del saber, desarrolla nuevas y mejores estrategias de aprendizaje, formula teorías, ensaya prácticas más eficientes que las anteriores y profundiza con más claridad en la comprensión ontológica de las causas y de los efectos, que es en sí misma una filosofía sobre la innovación (Seltzer y Bentley, 2000).

Pero paradójicamente el ser humano, al tiempo que ha ganado en bienestar, en conciencia de sí mismo, en capacidad de entendimiento y en libertad de acción, ha construido un hábitat adverso a su propia naturaleza. Las razones y sin razones de la edad moderna, se han constituido en un hábitat global contrario a la convivencia humana (Seltzer y Bentley, 2000). Las personas no hemos aprendido aún a convivir en sociedad y a cuidar de la tierra, la gran casa que habitamos. La innovación se convirtió en consumismo y en guerras de mercado, cuando de ella ha debido surgir el bienestar de la cultura con calidez humana.

Es que el homo faber, el referido antes, es también un gran depredador, un destructor incontenible, más que cualquier otro animal sobre la tierra. Ha sido capaz de transformar todo lo que le rodea, para bien o para mal, y no ha sido capaz de innovar radicalmente sobre su propia convivencia.

Del homo faber, de este homo con capacidad de actuar y de fabricar cosas, tendrá que salir el homo solidario, con nivel de conciencia superior, como el mejor modelo de innovación evolutiva. Un homo solidario con rectitud ética y sólida moralidad social, que sea capaz de cultivar su propia cultura solidaria (Zabala, 1998). Esta visión futura de utopía, no puede perderse de vista, porque el surgimiento del homo solidario - y estamos ad portas de ello-será el despertar y el comienzo de una nueva humanidad.

\section{Destrucción creadora y cultura de la innovación}

Entre todas las invenciones, sobresalen los sistemas socioeconómicos que el ser humano ha creado para desarrollar sus diferentes modelos de cultura y sociedad. Hace pocos miles de años llegaron a su fin el modelo prehistórico y el modelo esclavista, hace breves siglos el modelo feudal y hace apenas dos centurias y media, surgió el capitalismo. El sistema socialista no continuó más allá de los años sesenta.

Estos sistemas socioeconómicos no perduraron en la historia, al igual que sucede y sucederá con el capitalismo, por su carga de inequidad y de exclusión social. La humanidad no ha encontrado aún el sendero de su propio bienestar colectivo.

Schumpeter escribió hace cincuenta años:

El capitalismo es, por naturaleza, una forma o un método de innovación económica y no solamente no es jamás estacionario, sino que no puede serlo nunca [...] El impulso fundamental que pone en movimiento a la máquina capitalista procede de los nuevos bienes de consumo, de los nuevos métodos de producción y transporte, de los nuevos mercados, de las nuevas formas de organización industrial que crea la empresa capitalista [...] [Los empresarios capitalistas] revolucionan de manera incesante la estructura económica desde dentro, destruyendo sin interrupción lo antiguo y creando continuamente elementos nuevos. Este proceso de destrucción creadora constituye una característica esencial del capitalismo. En ella consiste en definitiva el capitalismo y toda empresa capitalista tiene que amoldarse a ella para vivir. (Capitalismo, socialismo y democracia, 1983, p. 95).

Pero la realidad es que hoy día los grandes dueños del capital siguen siendo un pequeño círculo privilegiado y los pobres en todos los pueblos de la tierra son apenas una masa incontable que subsiste con mínimos vitales (Krugman, 2012). Ni la producción de los bienes y servicios es suficiente ni su distribución es adecuada ni el consumo de los mismos responde a las necesidades reales de los ciudadanos (Amartya, 2000).

La acumulación del capital es de unas proporciones tales (Krugman, 2012), que parece estar cercano el día en que no necesitará más de las personas para reproducirse y manejará la vida de todos los seres humanos a su antojo, como una medusa gigante superior a todo cuanto existe. Y más ahora en el mundo de la virtualidad, cuando este ser de mil cabezas, de abismales proporciones, pasó a ser un agitado cúmulo de luces movido de manera incesante por las interfaces agitadas de los computadores y de los artefactos electrónicos.

Es imperativo entonces, aplicar ahora más que nunca una destrucción creativa (Schumpeter, 1983) 
mediante la gestión de la innovación social, en relación con las distorsiones de un sistema capitalista que se hace cada vez menos viable para la equidad, más injusto y más sujeto a crisis recurrentes (Benerjee y Duflo, 2012). El ser humano está cada vez más urgido de superar la profunda angustia que le genera no encontrar un mundo mejor para mejor vivir (Amartya, 1999). Un camino cierto es la innovación social a través de la cultura solidaria innovadora.

Particularmente, son palpables los vertiginosos cambios en las tecnologías de información y comunicación de los últimos años a nivel mundial. Basta mirar también la innovación en los campos de la biotecnología, la robótica, las fuentes de energía, la cibernética, los materiales para construcción, la nanotecnología, la física cuántica, la química inorgánica, la astrofísica, por mencionar solo algunos (Brooks, 1982). Sin embargo, hay gran diferencia en los procesos de innovación al observar los cambios en las prácticas culturales, en los niveles de conocimiento, en el impacto de la educación sobre la sociedad o en los efectos de los cambios generacionales sobre la conducta humana.

\section{La paradoja de la solidaridad}

La cooperación recíproca, así como la cultura solidaria y la innovación social (Álvarez, 2011), se convierten en una paradoja cuando apuntalan el progreso de los pueblos o cuando contribuyen a todo lo contrario. Esto es similar en las instituciones sociales. Las guerras son ejemplo de cooperación nefasta al interior de cada una de las partes en conflicto. Para vencer al enemigo la contraparte debe estar unida, de lo contrario nunca vencerá. Es que hay solidaridad entre los que hacen el bien y solidaridad entre los que hacen el mal. Como también hay cooperación entre los que buscan el bien común y entre los que fabrican el delito. Su diferencia está en el propósito y en los fines perseguidos. Los unos y los otros se nutren de la solidaridad, solo que el resultado de sus acciones es mutuamente excluyente (D’Inrivarne, 1987). El método no hace la diferencia; el resultado sí. La innovación se torna una paradoja según sea el objetivo al que se aplique.

Algo esencial debe fundamentar la gestión de la innovación social en relación con el carácter de identidad sobre la cultura solidaria dentro de una organización empresarial colectiva. Dado que la filosofía de asociación humanística, es característica esencial en una cooperativa, tal vez sea este el aspecto diferenciador en el contexto de la sociedad respecto a otras organizaciones y sobre todo entre los diferentes modelos de gestión empresarial (Zabala, 1998). Esa diferencia es la que debe nuclear con identidad a quienes están vinculados o desean pertenecer a una empresa cooperativa (Pineda, 1999). Su filosofía humanística da identidad a la cultura solidaria que los une en torno a su accionar colectivo. Y aquí comienza a surgir una nueva faceta en la gestión de la innovación social para superar el conflicto que genera la paradoja de la solidaridad.

\section{Cultura de la solidaridad innovadora}

El término cultura proviene del latín cultus, que significa 'cultivar'. En la cultura se cultiva el espíritu humano, se cultiva la mente y la inteligencia, las buenas o las malas prácticas. Por eso la cultura se refiere al conjunto de experiencias, conductas, costumbres, saberes, criterios, conocimientos o ideas que caracterizan a una sociedad. La cultura es un proceso dinámico, adaptativo y se renueva generalmente con referentes del entorno en un ambiente simbiótico de aprendizaje continuo (Weber, 2011). La cultura se enriquece con procesos educativos, participativos o vivenciales y a través de ellos es posible adquirir nuevos aprendizajes y también cambiar conductas, principios, costumbres, tradiciones, conocimientos, valores, creencias o paradigmas (Radkau, 2011). Esta cultura, concebida así, pasaría a ser objeto de la innovación social.

A la luz de lo anterior, en el marco más amplio de la cultura en general, es posible concebir la cultura solidaria innovadora como el cultivo permanente de las prácticas solidarias y de la ayuda recíproca entre los seres humanos con fines de bienestar común y de gestación cotidiana de su propia felicidad grupal. La solidaridad asociativa debe tener un sentido (Zabala, 1998), es decir, un propósito y en este caso es el bien común de los individuos que se unen para ser más fuertes y ser más en su vitalidad, mediante la ayuda mutua (Ramírez, 1989). El individuo aislado es frágil; por el contrario, el grupo unido será sólido y fuerte (Uribe, 1993).

Componente esencial de la cultura solidaria es la filosofía del dar y recibir que es la base de la ayuda mutua, del "apoyémonos en nosotros mismos", que en eso consiste la autoayuda, en la que todos somos importantes, todos valemos. Esa es la fuerza de la participación (Böök, 1992). Esta filosofía es solidaria porque eleva la altura moral de las personas, porque las 
dignifica, las hace mejores, dado que hay apoyo recíproco entre todos, sin exclusión alguna (Uribe, 1998). Hay por tanto, causa sensitiva frente a las dificultades de los demás, compromiso de conciencia colectiva, es decir, de solución permanente a los problemas comunes de la cooperación asociativa (Watkins, 1973). El que más tiene, más puede dar a los que menos tienen y eso es equidad. Esta es en esencia, la filosofía cooperativa y por eso el cooperativismo es ante todo, cultura solidaria (ACI, 1987). El reto es cómo cualificar esta cultura solidaria mediante la gestión de procesos de innovación social.

La innovación social es un permanente desafío en el desarrollo de las sociedades que buscan hacerse sostenibles (Dávila, 2004). Por el camino de la innovación es como se ha mejorado siempre el progreso de cualquier sociedad. La capacidad de innovar es un proceso creativo. Toda persona es capaz de ser creativa a su modo. Por eso se afirma que la innovación social es un proceso de emprendimiento individual y lo puede ser también de carácter colectivo, cuyo propósito es generar nuevas prácticas sociales, nuevas soluciones que aporten al bienestar de todos los miembros de la sociedad y esto se va alcanzando a medida que logra difundirse el resultado del proceso innovador (Aristizabal, 2012).

Tanto las innovaciones técnicas como las innovaciones sociales, que surgen de la creatividad individual o colectiva, se orientan a buscar soluciones a problemas y desafíos de la sociedad. Por esta razón algunos consideran la propuesta cooperativa como una innovación social (Dávila, 2004), porque su propósito y su forma, siendo al mismo tiempo empresa económica y asociación de personas, se constituye en un modelo de organización cuya ventaja estratégica permitirá resolver de manera eficaz los múltiples problemas económicos, sociales, culturales y ambientales de las personas que se unieron para ayudarse entre sí (Sanchis y Campos, 2008).

Lida Esperanza Villa Castaño afirma:

Aunque el concepto de innovación social es relativamente reciente, la literatura es abundante. Desde diferentes perspectivas disciplinares como las ciencias políticas, la administración y la economía, el concepto se relaciona con la necesidad de transformar una realidad o una problemática social como por ejemplo la pobreza, la violencia, la exclusión o, dicho en términos concretos, la ausencia de bienestar.
Frente a estos problemas puntuales en la contemporaneidad, la innovación social brota como una posibilidad por los distintos actores y sectores, de dar soluciones novedosas frente a problemas concretos. (2014, p. 31).

Al gestionar la innovación social se plantean soluciones nuevas, es decir, innovadoras y creativas, en relación con problemas específicos que aquejan a cualquier comunidad de personas. Sus desafíos comprenden la pobreza, el hambre, el desempleo, la falta de educación y conocimiento (Guerra, 2014), el acceso a la tecnología, al mundo digital, al uso de energías limpias, a producciones técnicas de baja escala, a la preservación del medio ambiente y sobre todo a la preservación de las fuentes hídricas y a la construcción de hábitats donde se cultive la felicidad de las familias (Amartya, 2000).

Por lo motivos anteriores se confirma cada vez más que es necesario aprender a gestionar la innovación social. Han sido muy claras las metodologías para hacer gestión de la innovación tecnológica, sobre todo en el ámbito empresarial y en algunos aspectos de las políticas públicas (Guerra, 2006). Sin embargo, esas mismas metodologías no son siempre adecuadas para hacer gestión de la innovación social (Köhler, 2000). Tal vez en el enfoque conceptual, en la práctica pedagógica o en la metodología de investigación adecuada, estará el arte de la gestión para construir innovación social.

Tendría gran sentido pensar desde el mundo académico, desde las empresas productivas, desde los centros de investigación, desde las políticas públicas (IEMP, 2011), o desde la difusión de los medios informativos, en un nuevo modelo de organización empresarial para gestionar desde esta, la innovación social que requiere el mundo actual. Quizás no sea la estructura cooperativa, sino organizaciones de tipo colaborativo. Cabe también en esta sinergia de talento humano, ocuparse de cómo hacer gestión eficaz de la innovación social, no solo en un ambiente local comunitario, sino en el contexto de un nuevo orden económico nacional e internacional (Monsalve, 2014).

Si la gestión administrativa integra recursos técnicos y físicos y la gestión financiera integra recursos de capital en el corto y largo plazo, la gestión social integrará el talento de las personas, el conocimiento, la creatividad, la experiencia, los espacios sociales, la cultura viva y ella será el terreno abonado para impulsar la innovación social (Dávila, 2013). Así, la gestión social será sinergia innovadora en función del bienestar humano. 


\section{Innovación social creadora de valor}

Los teóricos del tema conciben la innovación social como un proceso para crear valor en beneficio de la sociedad. Esta creación de valor incluye buenas prácticas (Dávila, 2013), modelos de gestión, novedosos productos o servicios, enfoques diferentes sobre la acción social y mejoras sustanciales en la estructura del capital social. La innovación social debe satisfacer necesidades concretas de los seres humanos, resolver problemas con mayor eficacia que las soluciones existentes y producir cambios favorables en los sistemas culturales o sociales en los que conviven las personas (Prevost y Roy, 2007).

Hace algunos años, el Gobierno de Estados Unidos solicitó a un grupo de expertos definir en qué consiste la innovación y este fue el resultado:

Innovación es el diseño, invención, desarrollo y/o implementación de productos, servicios, procesos, sistemas, estructuras organizacionales, o modelos de negocio, nuevos o modificados, con el propósito de crear un nuevo valor para los consumidores y un retorno financiero para la empresa (Senge, 1992, p. 387).

Esta es casi una definición de mercadeo, porque prescinde del sentido de bienestar social sin fines lucrativos que sería el objeto de la innovación social.

La gestión eficaz de la innovación social debe orientarse a incrementar el potencial de escalabilidad y replicabilidad que debe tener toda idea innovadora y además asegurar la sostenibilidad de cualquier proyecto social novedoso, sobre todo con la participación (Ramírez, 2012) y el empoderamiento de la comunidad beneficiada. Si la gestión no lograr estos propósitos, no tendrá el impacto necesario para mantener en pie la idea innovadora (Prahalad, 2010).

\section{La innovación social es un proceso}

En los conocidos mapas de valor no lineales ${ }^{1}$ sobre la gestión de la innovación social, se reconocen seis etapas de gran importancia (Seltzer et al., 2000) que deben ser gestionadas con rigor metodológico, aunque no siempre secuenciales. En la tabla 1 se

1 En matemáticas existe el concepto de "sistemas dinámicos planos" que incluyen el de" sistemas no lineales", que por su naturaleza tienden a ser inestables y el cálculo de su solución es impredecible. observa cada una de las etapas que se deben tener en cuenta en un proceso de innovación social.

Tabla 1

Etapas para llevar a cabo un proceso de innovación social

\begin{tabular}{lll}
\hline 1 & $\begin{array}{l}\text { Retos y oportu- } \\
\text { nidades }\end{array}$ & $\begin{array}{l}\text { Claridad sobre los desafíos } \\
\text { implícitos en cualquier pro- } \\
\text { blema de índole social }\end{array}$ \\
\hline 2 & Alternativas & $\begin{array}{l}\text { Ideas y propuestas que } \\
\text { deben ser analizadas por los } \\
\text { beneficiarios de la innovación } \\
\text { social. }\end{array}$ \\
\hline 3 & Experimentación & $\begin{array}{l}\text { Que propicia el cotejo entre la } \\
\text { teoría y la práctica para hacer } \\
\text { las correcciones necesarias. }\end{array}$ \\
\hline 4 & Sostenibilidad & $\begin{array}{l}\text { Aseguramiento en relación } \\
\text { con la idea innovadora, } \\
\text { es decir, la capacitad de } \\
\text { mantenerse en el tiempo y } \\
\text { de dar apoyo a otras ideas } \\
\text { relacionadas. }\end{array}$ \\
\hline 5 & Escalabilidad & $\begin{array}{l}\text { De la idea innovadora, para } \\
\text { lograr cada vez más benefi- } \\
\text { cios amplios e incluyentes. }\end{array}$ \\
\hline & & $\begin{array}{l}\text { En el sistema social o eco- } \\
\text { nómico donde se desarro- } \\
\text { llará el modelo de innova- } \\
\text { ción social propuesto. }\end{array}$ \\
\hline & &
\end{tabular}

Nota. Elaboración del autor con base en los mapas de valor no lineales.

La innovación es un proceso continuo, tal vez de nunca acabar, porque estando su origen en la mente humana, resulta inagotable su capacidad de imaginar, de crear nuevas realidades y de perfeccionar sus propias invenciones.

En matemáticas la resolución de una ecuación no lineal se hace por aproximaciones sucesivas. Las intersecciones de las curvas y rectas en su representación geométrica, se asemejan a una telaraña que "acorrala" un punto focal determinado. No es fácil representar el caos mediante cálculos matemáticos, como tampoco lo es calcular los movimientos de las olas o las oscilaciones de un péndulo en situación inestable. La innovación social es parecida a este enfoque que acaba de expresarse: su desenvolvimiento es un proceso continuo de nunca acabar y sus avances se darán según sea la dinámica cultural de una sociedad.

En la figura 1 aparece una amonita al lado izquierdo y al derecho su representación geométrica no lineal, según la percepción de Galileo Galilei sobre las 
"curvas de la vida". Según este eminente hombre del Renacimiento, "el libro de la naturaleza" está escrito en lenguaje matemático. Las espiras de la concha de la amonita aumentan su anchura de acuerdo con un factor constante. Para Leonardo da Vinci, estas criaturas expanden su casa y su techo gradualmente, en proporción, conforme crece su cuerpo manteniéndose pegado a los lados de la concha. Esta fue también, muchos años antes, la propiedad que caracterizara a la espiral logarítmica descubierta por Arquímedes ${ }^{2}$ en la Grecia antigua.

Las ideas, propuestas, ensayos y proyectos, se van resolviendo en un continuo no lineal, ascendente, alrededor de un punto inicial, en una especie de espiral, a medida que surgen nuevas realidades y necesidades en el desarrollo de un grupo humano o de una comunidad específica.

En el proceso de la innovación social la comunidad que participa es un actor clave y lo es también el innovador social, que lidera y emprende, al igual que los diferentes actores de soporte administrativo, financiero, político o científico (Valdés, 2008). Pero para ello es indispensable un medio propicio con el fin de alcanzar el éxito de la innovación: la amplia difusión social de la idea innovadora a partir de un punto focal.

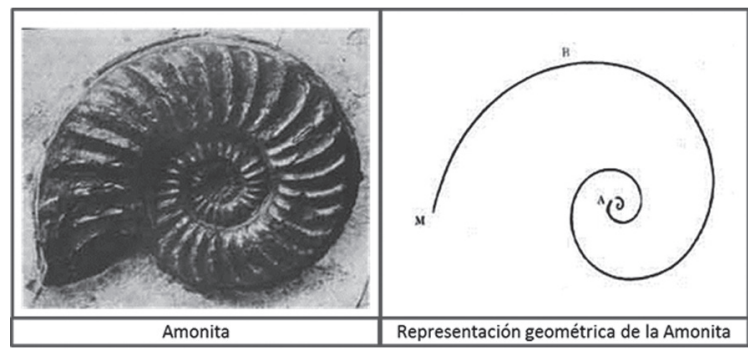

Figura 1. Representación geométrica no lineal de una amonita, según Galileo. Departamento de Estadística e Investigación operativa de la Universidad de Alicante, España.

\section{Gestores de innovación social}

El Manual de Oslo, en su versión del $2006^{3}$, define cuatro tipos de innovaciones: productos, procesos,

\footnotetext{
2 Concepto tomado de la página web de la Universidad de Alicante, España: http://deio.ua.es/es/licenciatura-matematicas/comentarios-al-plan-de-estudios/comentarios-al-plan-de-estudios. html

3 El Manual de Oslo es una guía para recoger e interpretar datos sobre la innovación, publicado por la ocDE y Erustat. Anterior a este el Manual de Frascati sobre investigación y desarrollo experimental. El Manual de Canberra enfatizó la demanda de recursos humanos para ciencia y tecnología.
}

mercadeo y organización (Villa, 2014). Se visualiza la innovación como un proceso en red, con múltiples actores que generan al mismo tiempo nuevos conocimientos y nuevas tecnologías. Entre los actores están los centros de investigación, las instituciones de educación superior, las entidades del sector público, las empresas privadas, las organizaciones no lucrativas y los organismos internacionales. Pero el manual apunta a la innovación tecnológica y más bien poco a la innovación social.

Se requieren gestores de innovación social para reducir la pobreza, resolver los graves problemas de seguridad alimentaria, superar las deficiencias en salubridad social, generar empleo, o implementar tecnologías limpias y mitigar el cambio climático (Villa, 2014).

Se requieren también gestores de innovación social (Villa, 2014) para lograr un más fácil acceso a la educación de las personas, disminuir la violencia en todas sus formas, favorecer el emprendimiento entre los jóvenes, proteger a los ancianos y a los niños, crear cultura solidaria unida al cuidado de los hábitat humanos, avanzar en la equidad de género, respetar a las minorías o abrir espacios viables a la inclusión social.

Si a nivel global se difundiera una cultura socialmente innovadora, gran parte de las necesidades y de las problemáticas sociales podrían resolverse de manera más eficaz que en la realidad actual. La pedagogía cívica podría transformar una gran ciudad, o el deporte entre barriadas, o acabaría con los conflictos entre pandillas de jóvenes.

En el escenario de lo social, hay un camino nuevo, inexplorado aún, para abordar soluciones de manera participativa: la economía social y solidaria, la socioeconomía de la solidaridad (Guerra, 2014). Podría ser un modelo innovador si se aplicara en gran escala. Es una economía basada en la propiedad solidaria, no en la propiedad pública, ni en la propiedad privada (Quijano y Reyes, 2004). No será capitalismo, no será socialismo, no será la actual economía de mercado. Será una economía del bienestar (Amartya, 2000), una economía de la felicidad, diferente a lo actual, donde el ser humano sea el eje y el propósito de todas las acciones económicas.

A través de una nueva cultura solidaria, que debe incluir educación (Silva, 2009), información, comunicación, divulgación, investigación y gestión de la experiencia acumulada, es posible alcanzar altos niveles de innovación social. ¿De qué manera? Produciendo realidades nuevas y mejorándolas a partir de las ya existentes, interactuando con otros 
entornos culturales que hayan desarrollado las mejores prácticas sociales (Vigostsky, 1978) ${ }^{4}$ o desarrollando una gran capacidad de aprendizaje autocrítico sobre las propias experiencias.

El ser humano, con su asombroso potencial adaptativo para transformar la realidad, es capaz también de reinventar su cultura social mediante nuevas prácticas, nuevos comportamientos, nuevas conductas y costumbres (Jaramillo, 2007). La realidad transforma la mente; de igual manera las nuevas prácticas y los nuevos aprendizajes. A través del actuar, las personas cambian su forma de pensar. Este debe ser un desafío en cualquier cooperativa: transformar su propia cultura asociativa en cultura solidaria innovadora sostenible.

\section{Compromiso empresarial cooperativo}

Es preocupante observar en muchas cooperativas cómo se promueve la incultura de la solidaridad favoreciendo prácticas individualistas que solo buscan la satisfacción individual o la complacencia del egoísmo propio, desde la gobernanza de los Consejos o desde la gestión administrativa (Mansilla, 2003). En donde prime ante todo la cultura insolidaria, jamás podrá existir cooperativismo sostenible (Arango, 2005). Entre muchos asociados prima siempre la relación monetaria con su cooperativa, en sustitución de la relación solidaria que es lo esencial en el acuerdo cooperativo. En donde el "yo" de cada quien esté por encima del "nosotros", nunca se podrá construir verdadera cultura asociativa (Monsalve, 2014).

Es posible convertir la cultura solidaria al interior del cooperativismo (Rojas et al., 2007) en una verdadera innovación social. Para ello se requiere de un nuevo liderazgo emprendedor entre quienes dirigen las cooperativas. Una cooperativa bien dirigida, bien administrada, exitosa, competente, en donde se practiquen a cabalidad los principios del cooperativismo (Uribe, 2012) puede ser igualmente un modelo de innovación social capaz de generar poder colectivo de alta transformación social, y resolver así problemas sociales, económicos, culturales y medioambientales de cualquier comunidad (Mansilla, 2003). De este

4 Este destacado investigador científico (1896-1934), ruso fundador de la psicología histórico-cultural, dio origen al término "Zona de desarrollo próximo" (ZDP) refiriéndose al espacio o brecha entre las habilidades que ya posee el/la niño/a y lo que puede llegar a aprender a través de la guía o apoyo que le puede proporcionar un adulto o un par más competente. Este concepto se podría aplicar a contextos de cultura local. modo, gestionar los destinos de una cooperativa, sería una buena forma de gestionar un modelo de innovación social.

Gestionar la innovación social es gestionar la igualdad social (Stiglitz, 2012), el acceso a la educación y al empleo, la superación de la pobreza, las oportunidades para vivir mejor, la responsabilidad social, la colaboración solidaria, el desarrollo sostenible, las nuevas iniciativas de innovación social, la preservación de los sistemas ecológicos, la seguridad social para todos y la construcción de una sociedad más justa.

Un desafío de las instituciones educativas, de las empresas actuales y de los entes gubernamentales es formar gestores para desarrollar este nuevo modelo, que podría denominarse "socioeconomía del bienestar solidario" (Silva, 2009) ${ }^{5}$. Se trata de construir la cultura social innovadora de la solidaridad asociativa, entendida esta como el conjunto de nuevos valores, normas, creencias, ideologías, hábitos, costumbres y códigos de conducta compartidos, para sustentar los nuevos procesos de la equidad social (Guerra, 2014). La construcción de una nueva cultura solidaria innovadora, debería ser el motor de la innovación social cooperativa basada en su gran ventaja estratégica asociativa.

El mundo entero requiere con urgencia la articulación de actores sociales que generen soluciones innovadoras, pertinentes, sostenibles, y escalables para el mejoramiento de la calidad de vida de la población en situación de pobreza extrema (Polanyi, 1989). Si a nivel global se difundiera una cultura socialmente innovadora, gran parte de las necesidades y de las problemáticas sociales quedarían resueltas.

Gestionar la innovación social implica liderar procesos orientados a organizar y dirigir los recursos disponibles tanto humanos como técnicos y económicos, para aumentar la creación de nuevos conocimientos y su aplicación en el tejido social (Quijano y Reyes, 2004). En este sentido, podría suponerse que es necesario para ello, gestionar el sistema de innovación social, que incluiría a su vez cuatro entornos identificables: la tecnología centrada en el bienestar social, la antropología económica, el consumo racional y responsable y un sistema financiero incluyente (Stiglitz, 2012).

\footnotetext{
$5 \quad$ En Europa se ha desarrollado ampliamente una concepción sobre economía social, cuyo enfoque puede diferir del concepto más suramericano sobre economía social y solidaria o socioeconomía de la solidaridad, además de muchas otras denominaciones que se plantean a veces con algún grado de sesgo ideológico.
} 
Un sistema de innovación social aplicado por entero a mejorar la calidad de vida de todos los ciudadanos en el mundo.

Así como la gestión tecnológica es conocimiento y es práctica, así la gestión de la innovación social es conocimiento de la condición humana y es la práctica de la cultura solidaria, la cultura de la cooperación asociativa, sin la cual no podremos subsistir en el futuro.

Gestionar la innovación social es gestionar la igualdad social, el acceso a la educación y al empleo, la superación de la pobreza, las oportunidades para vivir mejor, la responsabilidad social, la colaboración solidaria, el desarrollo sostenible, las nuevas iniciativas de innovación social, la preservación de los sistemas ecológicos, la seguridad social para todos y la construcción de una sociedad más justa (De la Vega, 2014).

Se necesitan miles de personas como administradoras del bienestar social, capaces de liderar y administrar procesos de innovación social. Se requieren más administradores de las ciencias sociales, más administradores de la solidaridad, más administradores de la equidad social (Guerra, 2006). Estos administradores construirán con muchos más, la cultura social innovadora de la solidaridad asociativa, entendida esta como el conjunto de nuevos valores, normas, creencias, ideologías, hábitos, costumbres y códigos de conducta compartidos para sustentar los nuevos procesos de la socioeconomía solidaria (Serrano, 2007).

En la utopía de la socioeconomía solidaria (Guerra, 2014), que debería ser la visión para Suramérica de la economía social al estilo europeo, vendría muy bien el sostenido espíritu emprendedor que significa la gestión permanente de la innovación social. En Colombia un joven innovador viene ofreciendo en algunas ciudades del país un litro de luz en una botella plástica con agua y cloro para tugurios sin electricidad. En la gestión de la innovación social, podría también ofrecerse un litro de solidaridad en el corazón de cada ser humano para construir un mundo más justo y venturoso.

\section{Conclusiones}

Es posible transformar la cultura solidaria de las organizaciones cooperativas a través de la innovación social. Antes de llevar a cabo actividades de educación cooperativa, es más importante fortalecer mediante la práctica, la autocrítica y la reflexión grupal, la conciencia solidaria de todos los miembros de una cooperativa.

La innovación social como experiencia creativa que busca resolver problemas y necesidades de la cooperación asociativa y de la relación de esta con las comunidades, es un camino por explorar para fortalecer la práctica de la cultura solidaria en las cooperativas.

La cultura solidaria innovadora en las empresas cooperativas, que son al mismo tiempo asociaciones de personas, podría convertirse en un gran modelo de innovación social para resolver los múltiples problemas económicos, sociales, culturales y ambientales de los grupos humanos que han puesto su esperanza en la ayuda mutua y en la fuerza de la cooperación asociativa. Esta nueva cultura, sería el componente básico de la gran ventaja estratégica cooperativa.

\section{Referencias}

Alianza Cooperativa Internacional. [ACI]. (1987). Las cooperativas en el año dos mil. Bogotá: Fondo Editorial Cincop Ltda.

Álvarez, J. F. (2011). Innovación y economía social y solidaria. Bogotá: Inversiones Ibarra Garrido. Recuperado de http://base.socioeco.org/docs/innovacion_y_ economia_social_y_solidaria.pdf

Amartya, S. (1999). Development as Freedom. New York: Knopf.

Amartya, S. (2000). Desarrollo y libertad (trads. E. Rabasco y L. Toharia). Barcelona: Planeta.

Arango, M. (2005). Manual de cooperativismo y economía solidaria. Medellín: Editorial Universidad Cooperativa de Colombia.

Aristizabal, J. D. (2012). Llenando espacios: un libro sobre emprendedores sociales. Bogotá: Editorial CESA.

Benerjee, A. V. y Duflo, E. (2012). Repensar la pobreza: un giro radical en la lucha contra la desigualdad global (trad. J. Mato). Bogotá: Aguilar.

Böök, S. A. (1992). Informe para el xxx Congreso de la ACI, Tokio. San José, Costa Rica: Oficina Regional de la ACI para Centro América y el Caribe.

Brooks, H. (1982). Social and technological innovation. En S. B. Lundstedt y E. W. Colglazier Jr. (eds.), Managing innovation (pp. 9-10). Elmsford, New York: Pergamon Press.

Dávila, R. (2004). Innovación y éxito en la gerencia cooperativa: casos exitosos de cooperativas rurales de ahorro $y$ crédito. Bogotá: Pontificia Universidad Javeriana. 
Dávila, R. (2013). Buenas prácticas cooperativas. Bogotá: Fondo Nacional Universitario y Centro de Investigación y Educación Cooperativa.

De la Vega, R. (2014). Innovación y emprendimiento social. Revista Javeriana, 810, 12-18.

Diaz, A. (2000). Luces y sombras en el cooperativismo colombiano. Bogotá: Instituto de Estudios Sociales Juan Pablo II.

D'Irivarne, A. (1987). Innovation technique et innovation sociale: les enjeux de la competitivité écononomique. Économíe et Humanisme, 294, 66-79.

Goleman, D., Kaufman, P. y Ray, M. (2000). The creative spirit. La revolución de la creatividad y cómo aplicarla a todas las actividades humanas. Buenos Aires: Ediciones $\mathrm{B}$.

Guerra, P. (2006). Las políticas públicas de empleo y el fomento de las economías solidarias en América Latina: Análisis de casos del Cono Sur. Cooperativismo \& Desarrollo, 18(96), 10-26.

Guerra, P. (2014). Socioeconomia de la solidaridad. Una teoría para dar cuenta de las experiencias sociales y económicas. Bogotá: Ediciones Universidad Cooperativa de Colombia.

Guiraud, P. (1996). La semiología. Madrid: Siglo Xxi Editores.

Jaramillo, J. (2007). Memorias intelectuales. Bogotá: Taurus, Santillana, Universidad de los Andes.

Krugman, P. (2012). Acabemos ya con la crisis (trads. C. Belza y G. García). Bogotá: Planeta Colombiana S. A.

Martínez, H. H. (2005). El marco ético de la responsabilidad social empresarial. Bogotá: Editorial Pontificia Universidad Javeriana.

Mansilla, M. A. (2003). Empresas cooperativas para una democracia económica. Bogotá: Centro de Investigación y Educación Cooperativa (CIEC)-Fondo Nacional Universitario de la Cooperativa de Profesores de la Universidad Nacional de Colombia.

Monsalve, Z. A. (2014). Cien claves de la economía solidaria. Bogotá: Centro de Investigación y Educación Cooperativa (CIEC)-Fondo Nacional Universitario de la Cooperativa de Profesores de la Universidad Nacional de Colombia.

Mora, C. (ed.) (2011). Políticas públicas y cooperativismo en Colombia: 30 años de encuentros y desencuentros. Bogotá: IEMP.

Pineda, C. J. (1999). Economía social o solidaria. Bogotá: McGraw-Hill.
Polanyi, K. (1989). La gran transformación: crítica del liberalismo económico (trad. J. Varela y F. Álvarez). Madrid: Ediciones Edymion C/Cruz Verde.

Prahalad, C. K. (2010). La nueva oportunidad de negocios en la base de la pirámide (trad. A. Buitrago). Bogotá: Grupo Editorial Norma.

Prévost, P. y Roy, M. (2007). La investigación sobre gestión de cooperativas: algunas reflexiones fundamentales. Revista de UniRcoop, 5(1), 231-254.

Quijano, J. E. y Reyes, J. M. (2004). Historia y doctrina de la cooperación. Bogotá: Editorial Universidad Cooperativa de Colombia.

Radkau, J. (2011). Max Weber, la pasión del pensamiento (trad. E. Webels). México: Fondo de Cultura Económica.

Ramírez, B. (1989). Teoría y doctrina de la cooperación. Bogotá: Escuela Superior Administrativa (Esacoop).

Ramírez, B. (2012). La cooperación, entre realidad y ficción. Revista Cooperativismo \& Desarrollo, 20(100), 131147.

Rojas, J., Bastidas, O., Gómez, P., Isola, G., Martín, A., Peixoto, P. y Silva, J. (2007). El paradigma cooperativo en la encrucijada del siglo XXI. Canadá: Universidad de Sherbrooke, Irecus.

Sanchis, J. R. y Campos, V. (2008). La innovación social en la empresa. El caso de las cooperativas y de las empresas de economía social en España. Economía e Industria, $368,187-196$.

Seltzer, K. y Bentley, T. (2000). The creative age. Knowledge and skills for the new economy. Madrid: Palgraphic. S.A.

Senge, P. M. (1992). La quinta disciplina. El arte y la práctica de la organización abierta al aprendizaje (trad. C. Gardini). Barcelona: Granica S.A.

Serrano, R. (2007). Apuntes críticos sobre democracia cooperativa. Revista Cooperativismo \& Desarrollo, 95, 6379.

Silva, J. A. (2009). Cooperación y solidaridad económica. Otra economía es posible. Revista Cooperativismo \& Desarrollo, 95, 137.

Stiglitz, J. E. (2012). El precio de la desigualdad. El 1\% de la población tiene lo que el 99\% necesita (trad. A. Pradera). Bogotá: Taurus.

Schnarch, A. (2006). Creatividad aplicada. Más allá de la información y el conocimiento. Bogotá: Arfo Editores e Impresores.

Schumpeter, J. A. (1985). Capitalismo, socialismo y democracia. Barcelona: Ediciones Orbis, S. A. 
Uribe, C. (1993). Bases del cooperativismo (4a ed.). Bogotá: Fondo Nacional Universitario.

Uribe, C. (1998). Crisis ideológica del cooperativismo, a la luz del pensamiento de Alexander Fraser Laidlaw. Bogotá: Fondo Nacional Universitario.

Uribe, C. (2012). Principios cooperativos. Bogotá: Centro de Investigación y Educación Cooperativa (CIEC)-Fondo Nacional Universitario de la Cooperativa de Profesores de la Universidad Nacional de Colombia.

Valdés, C. L. (2008). Los nuevos emprendedores. Revista Poder, 82-93.

Vélez, A. y Vélez J. D. (2010). Pensamiento creativo. Bogotá: Villas Editores.
Villa, L. E. (2014). Notas en torno al concepto de innovación social desde la perspectiva de las empresas. Revista Javeriana, 820, 30-35.

Vygotsky, L. S. (1978). Mind in Society. Cambridge, MA: Harvard University Press.

Watkins, W. P. (1973). La Alianza Cooperativa Internacional 1895-1970 (trads. M. A. Angueira y J. Ludmer. Buenos Aires: Intercoop Editorial Cooperativa Limitada.

Weber, M. (2011). La pasión del pensamiento. (1a ed.). México: Fondo de Cultura Económica.

Zabala, H. (1998). Las teorías sobre la solidaridad y el porvenir de la cooperación. Medellín: Editorial Latino. 